\title{
Calidad de cuidado materno: una comparación entre bebés prematuros en cuidado madre canguro y bebés a término en cuidado regular
}

\author{
Quality of Maternal Care: A Comparison of Preterm Infants in Kangaroo \\ Mother Care and Full-Term Infants in Regular Care
}

Recibido: febrero 2 de 2010 | Revisado: mayo 5 de 2010 | Aceptado: mayo 16 de 2010

\author{
Olga Alicia Carbonell ** \\ SANDRA Juliana Plata \\ PaOla ANDRea PeÑa \\ Pontificia Universidad Javeriana, Bogotá, Colombia \\ MARTHA CRISTO ${ }^{* * *}$ \\ Programa Madre Canguro Integral, Fundación Canguro, \\ Bogotá, Colombia \\ GERMÁN POSADA ${ }^{* * * * *}$ \\ Purdue University, Indiana, Estados Unidos
}

Para citar este artículo. Carbonell, O.A., Plata, S.J., Peña, P.A., Cristo, M. \& Posada, G. (2010). Calidad de cuidado materno: una comparación entre bebés prematuros en cuidado madre canguro y bebés a término en cuidado regular. Universitas Psychologica, 9 (3), 773-785.

* Artículo de investigación. Proyecto de investigación 00064: Contacto temprano piel a piel en madres adolescentes-niño/a-prematuro/a: Sensibilidad materna y comportamiento de base segura. Co-financiado por el Center for Mental Health Promotion de New York y Vicerrectoría Académica - Pontificia Universidad Javeriana (2005-2008).

** Facultad de Psicología, Cr. 5 \# 39-00 Piso 2. Correos electrónicos: carbonel@javeriana.edu.co ; sandra. plata@javeriana.edu.co; paolaplus@hotmail.com

****Correo electrónico: martha_cristo@yahoo.com

***** Correo electrónico: gposada@purdue.edu

\section{RESUMEN}

El propósito de esta investigación fue estudiar los efectos de la intervención del Cuidado Madre Canguro (CMC) en la sensibilidad materna, en una muestra de madres adolescentes con alto riesgo psicosocial. Se compararon dos grupos de díadas: madres adolescentes con bebés prematuros en cuidado madre canguro, y madres adolescentes con bebés a término en cuidado regular. Se realizaron observaciones en el medio familiar, para evaluar la calidad del cuidado materno. Los análisis indican que no existen diferencias significativas entre los dos grupos de madres adolescentes respecto a la calidad de cuidado materno. Este hallazgo permite suponer un efecto protector por parte del CMC en las díadas madres adolescente-bebé prematuros, ya que esta condición del bebé es un factor de riesgo adicional a las condiciones del grupo a término en cuidado regular. Estos resultados preliminares plantean la necesidad de continuar explorando los efectos de la intervención canguro en la sensibilidad materna, especialmente en poblaciones vulnerables.

\section{Palabras clave autores}

Sensibilidad materna, prematurez, embarazo adolescente, cuidado madre canguro.

Palabras clave descriptor

Método madre canguro, embarazo en adolescentes, relaciones madre-hijo.

\section{A B S T R A C T}

The purpose of this study was to examine the potential impact of an intervention program (Kangaroo Mother Care, $\mathrm{KMC}$ ) on maternal sensitivity in a sample of high-risk adolescent mothers. Two mother-infant groups were compared: adolescent mothers with their preterm baby in kangaroo care and adolescent mothers with their full-term baby in regular care. Naturalistic observations at the home environment were conducted to assess maternal quality of care. No significant differences were found between both groups of dyads. Results are in line with the notion that KMC seems to play a protective role for adolescent mothers and their premature babies, given the additional risk factor of prematurity when compared to the full term group. These preliminary findings are stimulating and support further inquiry into 
the effects of KMC on maternal sensitivity particularly in high-risk populations.

Keywords authors

Maternal Sensitivity, Prematurity, Adolescent Pregnancy, Kangaroo Mother Care.

Keywords plus

Kangaroo Mother Method, Teenage Pregnancy, Mother-Child Relations.

El embarazo adolescente continua siendo una problemática psicosocial y de salud, a nivel mundial, que necesita ser comprendida e intervenida (Moore \& Brooks-Gunn, 2002; PubMed, 2003; WHO, 2009). A pesar de que las tasas han declinado en los países desarrollados, especialmente desde la década del 90 (Child Trends, 2004), los datos proporcionados por la Organización Mundial de la Salud (2009) indican que aproximadamente 16 millones de niñas adolescentes en el mundo están teniendo hijos cada año. Esto equivale al $11 \%$ de la tasa mundial de nacimientos. El 95\% de estos embarazos ocurren en los países en vías de desarrollo con ingresos medios y bajos, siendo la tasa de embarazo adolescente de éstos últimos, cinco veces más alta comparada con la de los países desarrollados.

Actualmente, en Latinoamérica y el Caribe, el embarazo adolescente representa un grave problema, que se ha ido incrementando sustancialmente (PubMed, 2003; Torres Ruiz, 2009). Colombia, entre el 2000 y 2006, ha mantenido una tasa de embarazo adolescente del $22 \%$ por cada 1000 adolescentes. Esta tasa es la más alta de Suramérica y quizá una de las más altas del mundo (Agencia Pandi, 2009; Profamilia, 2005). Las tasas más elevadas de embarazo adolescente se dan en los hogares de las zonas más pobres y en los grupos étnicos más desfavorecidos a nivel económico y educativo (Celsam, 2009; Darroch, Singh, Frost $\&$ the Study Team, 2001; Moore \& Brooks-Gunn, 2002; Profamilia, 2005; WHO, 2009).

El embarazo adolescente conlleva riesgos para la salud materna e infantil. Las adolescentes entre 15-19 años tienen mayor probabilidad de morir en el parto, y en madres adolescente muy jóvenes, menores de 14 años, el riesgo se incrementa (WHO, 2007, 2009). Así mismo, la prematurez y el bajo peso al nacer son factores de riesgo, especialmente en los países en desarrollo, y en el caso de las adolescentes embarazadas, éstas tienen una mayor probabilidad de tener bebés prematuros, y/o de bajo peso. Estos factores aumentan la posibilidad de muerte temprana del bebé y, a pesar de que en las últimas décadas se ha incrementado la supervivencia de los bebés prematuros, muchos de los supervivientes son más vulnerables a problemas de salud y desarrollo (Charpak, Ruiz-Peláez \& Figueroa de Calume, 1996; Charpak, Ruiz-Peláez, Figueroa de Calume \& Charpak, 2001; Child Trends Data Bank, 2008; Scholl, Hediger \& Belsky, 1994; WHO, 2009).

El embarazo precoz también genera altos costos sociales. La mayoría de las madres adolescentes abandonan sus estudios, truncando proyectos profesionales, aumentando y transmitiendo la pobreza de una generación a otra (Agencia Pandi, 2009; Moore \& Brooks-Gunn, 2002; WHO, 2009). Las madres adolescentes generalmente no están preparadas para asumir las responsabilidades económicas y los retos psicológicos y emocionales que conlleva la crianza temprana, pues, en la mayoría de los casos, estos embarazos ocurren por fuera de una relación matrimonial y por lo tanto, les toca asumir las responsabilidades de la maternidad solas, sin el apoyo de los padres de sus hijos o hijas (Child Trends Data Bank, 2004, 2008; Moore \& Brooks-Gunn, 2002).

Igualmente, las madres adolescentes son una población de alto riesgo psicosocial, dado que poseen menor conocimiento sobre desarrollo infantil, prácticas de crianza, muestran menor capacidad de regulación emocional en las interacciones con sus hijos/as y presentan mayor probabilidad de utilizar estrategias disciplinarias maltratantes (Haskett, Johnson \& Miller, 1994; Lutzker, Van Hasselt, Bigelow, Greene \& Kessler, 1998; Moore \& BrooksGunn, 2002; Stevens-Simon \& Nelligan, 1998). Las madres adolescentes con bajo nivel socioeconómico y bebés a término, tienden a caracterizarse por ser poco tolerantes, empáticas y comprensivas frente a las necesidades del bebé, a no establecer interacciones físicas cercanas, no respetarlo como individuo, desconocer su proceso de desarrollo y fomentar escasas habilidades verbales en éste (Ortiz, Borré, Carrillo \& Gutiérrez, 2006). 
Respecto a los bebés prematuros, diversos estudios muestran que existe mayor estrés en la relaciones padre/madre-bebé prematuro, puesto que son considerados por los padres como niños/as más difíciles, que muestran mayor afecto negativo y dificultad para regular sus emociones, y no son responsivos a las intervenciones de los padres para calmarlos, lograr su atención o interpretar con facilidad sus señales sociales. Esto exige que los padres inviertan mayores esfuerzos y tiempo, comparados con los bebés a término (Brachfeld, Goldberg \& Sloman, 1980; Moran, Pederson, Pettit \& Krupka, 1992; Pederson, Bento, Chance, Evans \& Fox, 1987; Spiker, Fergurson \& Brooks-Gunn, 1993).

\section{Programa Madre Canguro}

En 1978, en Bogotá-Colombia, se crea el programa madre canguro en el Instituto Materno Infantil, por parte del doctor Edgar Rey, para suplir la escasez de incubadoras y disminuir las infecciones nosocomiales debidas al hacinamiento de los servicios de neonatos (Figueroa de Calume \& Charpak, 1996). Este es un programa alternativo de cuidado para bebés prematuros menores de 37 semanas gestacionales y de bajo peso al nacer (inferior a $2.500 \mathrm{~g}$; Tessier et al., 1998), cuya tecnología es de alta calidad, relativo bajo costo y accesible en hospitales de primer, segundo y tercer nivel con recursos limitados, en países en vías de desarrollo (Cattaneo, Davanzo, Bergman \& Charpak, 1998). El programa madre canguro posee unos prerrequisitos de elegibilidad: al salir del hospital el bebé debe superar los 1.900 g, tener adecuada coordinación succión-deglución, ser capaz de ser amamantado, y no poseer ninguna patología. Así mismo, se requiere que la red de apoyo familiar siga las instrucciones del cuidado madre canguro (Figueroa de Calume \& Charpak, 1996).

El cuidado madre canguro posee tres componentes: 1) posición canguro: contacto piel a piel durante 24 horas al día, se coloca al bebé en posición vertical en el pecho de su madre, hasta que logre un peso de $2.400 \mathrm{~g}$. La madre le ayuda a regular la temperatura y la respiración; 2) nutrición canguro: lactancia exclusiva y leche de fórmula cuando el/la bebé lo necesita; 3) control clínico: asistir todos los días a control hospitalario hasta que el/la bebé, durante tres días consecutivos, suba de 20 a 30 gramos diarios. Después, las consultas son cada tres días, luego semanalmente y, para finalizar, éstas son mensuales (Charpak, Ruiz-Peláez \& Figueroa de Calume, 1996).

Estudios realizados sobre el cuidado madre canguro apoyan hallazgos iniciales respecto a sus efectos benéficos e indican que el cuidado madre canguro es comparable con el cuidado tradicional en incubadora en lo que concierne a la estabilidad fisiológica, reducción de la tasa de mortalidad y aumento en crecimiento del bebé prematuro. Además, las infecciones nosocomiales son menos frecuentes y la estadía en el hospital, más corta para los bebés canguro que para bebés en cuidado de incubadora (Cattaneo et al., 1998; Charpak, Ruiz-Peláez \& Figueroa de Calume, 2000; Charpak, Figueroa de Calume \& Ruiz-Peláez, 2000; Charpak, Ruiz-Peláez, Figueroa de Calume \& Charpak, 2001). No obstante, esta evidencia positiva en cuanto a aspectos fisiológicos del bebé prematuro, todavía se requiere investigar sobre efectos psicosociales de dicho programa, en términos del desarrollo infantil y de la calidad de las interacciones madre-hijo/a.

\section{Cuidado Madre Canguro y Efectos Psicosociales en el Desarrollo Infantil}

La evidencia preliminar existente indica que el cuidado madre canguro podría tener un impacto positivo en el desarrollo motor, cognitivo y perceptual de los niños, como lo muestran los puntajes del Bayley en los indicadores de desarrollo mental y psicomotor realizados a los 6 y 12 meses de edad corregida de los bebés prematuros (Feldman, Eidelman, Sirota \& Weller, 2002; Ogi et al., 2002). Así mismo, el estudio de Tessier et al. (2003) encuentra que al comparar los resultados en la prueba Griffiths a los 12 meses de edad corregida entre bebés prematuros de alto riesgo y de bajo peso que recibieron cuidado madre canguro y los de cuidado tradicional en incubadora, los primeros obtuvieron puntajes más altos en las subescalas de audición y habla, personal-social y ejecución. 
Igualmente, el contacto temprano piel a piel parece contribuir positivamente a la organización del sistema neurocomportamental y a la regulación emocional de los bebés prematuros comparados con los bebés con cuidado tradicional, en cuanto a mostrar ciclos de sueño-dormida más organizados, mejor regulación de emociones negativas y de sus estados emocionales, y presentar mayor atención sostenida y compartida con la madre al explorar objetos (Feldman, Weller, Sirota \& Eidelman, 2002; Ogi et al., 2002).

\section{Sensibilidad Materna y Cuidado Madre Canguro}

Los hallazgos preliminares respecto al impacto del cuidado madre canguro sobre las interacciones y relaciones madre-bebé, es menos clara y consistente. Tessier et al. (1998) reportan que existe un cambio positivo en las percepciones de las madres respecto a su competencia materna, cuando el tiempo de ingreso al cuidado madre canguro es más corto (durante los dos primeros días después del nacimiento) o cuando el estatus de salud del bebé es frágil y requiere cuidados intensivos, comparado con las madres cuyos hijos asisten al cuidado en incubadora. En estas dos situaciones, las madres participantes en cuidado madre canguro se perciben más sensibles y estimulan cognitivamente a sus bebés.

En un estudio posterior, Tessier et al. (2003) señalan que el cuidado madre canguro parece ser un medio para realzar la calidad de cuidado en el medio familiar, creando un clima donde las madres y los padres progresivamente se van volviendo más atentos respecto al hijo/a, y presentan mayor tendencia hacia un cuidado sensible. Igualmente, Ogi et al. (2002) afirman que el cuidado madre canguro promueve interacciones positivas entre el niño/a y sus padres. Anisfeld, Casper, Nozyce y Cunningham (1990) en un estudio experimental sobre el papel jugado en la responsividad materna al hijo/a al aumentar el contacto físico temprano mamá-bebé, a través de cargadores de tela a los 3.5 meses de edad, encontraron que éste promovía las respuestas maternas en muestras clínicas de madres de bajos recursos en su mayoría de origen latino y negro. Consistente con estos hallazgos, algunos estudios posteriores aportan evidencia respecto a un mayor cuidado sensible provisto por las madres, luego de la experiencia de cuidado intensivo piel a piel, en el programa madre canguro. Feldman et al. (2002) identificaron que tanto madres y padres fueron más sensibles y produjeron un mejor ambiente en el hogar, a diferencia del grupo de cuidado en incubadora, cuando sus bebés tuvieron 3 meses de edad. Igualmente, las madres de los bebés canguro fueron descritas como más sensibles, cálidas, adaptadas y recursivas en las interacciones sociales con sus bebés a los 6 meses, que las madres del cuidado en incubadora. Los resultados de Feldman, Weller, Sirota y Eidelman (2003) muestran que el cuidado madre canguro incrementa la sensibilidad materna y reduce el comportamiento intrusivo; las madres presentan mejor ajuste en las interacciones sociales mamá-bebé prematuro.

En una investigación reciente realizada en Bogotá, con población de madres adolescentes y adultas con bebés prematuros y/o de bajo peso que participaron en el programa madre canguro, comparadas con madres adolescentes y adultas con bebés a término con las mismas características sociodemográficas, los resultados mostraron que las madres adolescentes a término obtuvieron puntajes significativamente más bajos en sensibilidad materna, con respecto a los otros tres grupos de madres. Por el contrario, las madres adolescentes y adultas participantes en el programa madre canguro obtuvieron los puntajes significativamente más altos (Ortiz et al., 2006).

En resumen, la maternidad adolescente está asociada con riesgos biológicos y psicológicos tanto para las madres como para los bebés. Uno de dichos riesgos es el de la prematurez que hace aún más crítica la situación bio-psico-social para la díada mamá-bebé prematuro. Investigaciones iniciales parecen demostrar efectos fisiológicos y psicológicos positivos del cuidado madre canguro en el bebé. Respecto a las relaciones madre-bebé, la información existente es escasa, y prácticamente no se han realizado investigaciones en lo relacionado a la calidad de cuidado materno durante el primer año de vida del bebé en ambientes naturales. 
Así, este estudio indaga sobre la calidad de cuidado que madres adolescentes brindan a sus bebés en el contexto del hogar. Específicamente, la investigación indaga sobre si existen diferencias entre la sensibilidad materna de dos grupos de madres adolescentes de alto riesgo, uno con la característica de la prematurez del bebé y el otro con bebé a término. Ambos grupos poseen características demográficas con factores de riesgo asociados, tales como la edad, el estrato socioeconómico y el nivel educativo. El grupo de madres pertenecientes al cuidado madre canguro posee un factor estresor adicional, que es la prematurez de su bebé con todas las secuelas de salud y en el desarrollo social, emocional y cognitivo, que se han evidenciado en diversos estudios. No obstante, este grupo de madres también posee un factor potencialmente protector, ya que el cuidado madre canguro favorece una experiencia de contacto físico temprano, prolongado y piel a piel, que podría permitir a la madre desarrollar una mejor comunicación y conocimiento de su bebé.

\section{Objetivo}

Evaluar los efectos del cuidado temprano e intensivo piel a piel, en la sensibilidad materna, en una muestra de madres adolescentes de alto riesgo. Más específicamente, determinar si existen diferencias significativas respecto a la calidad del cuidado materno en cuanto a la sensibilidad materna y a las once subescalas del comportamiento materno, entre madres adolescentes con bebés prematuros y cuidado madre canguro, y madres adolescentes con bebés a término y cuidado regular.

\section{Método}

\section{Participantes}

La muestra estuvo conformada por 93 madres adolescentes, divididas en dos grupos, 49 madres con sus bebés prematuros y/o de bajo peso al nacer participantes en el cuidado madre canguro, y 44 madres con sus bebés a término, provenientes de los estratos socio-económicos 1 , 2, y 3 . La muestra de madres participantes fue voluntaria y recolectada en tres hospitales de primer nivel de la ciudad de Bogotá. Respecto al estado civil, dentro del grupo de madres con cuidado canguro, el 2.0\% estaban casadas, el 44.9\% en unión libre, el $18.4 \%$ en relación de pareja sin convivencia y el $34.7 \%$ sin relación de pareja. En cuanto al grupo de madres con cuidado regular, el 2.3\% estaban casadas, el 47.7\% convivía en unión libre, el 27.3\% mantenía una relación de pareja sin convivencia y el $22.7 \%$ sin relación de pareja. En cuanto a la ocupación actual, $33.3 \%$ de las madres del grupo canguro informaron que no estaban estudiando y $66.7 \%$ permanecía estudiando. En el grupo de madres de cuidado regular, el $54.5 \%$ no estudiaba y el 45.5\% sí lo hacía. El $87.5 \%$ de las madres canguros señalaron que no trabajaban y un $12.5 \%$ informó hacerlo. En el grupo de madres con cuidado regular, $70.5 \%$ no trabajaba y un $29.5 \%$ sí lo hacía. Datos demográficos adicionales de la muestra se presentan en las Tabla 1 y 2.

La prueba Chi-cuadrado mostró que no existen diferencias significativas entre los dos grupos de madres adolescentes participantes en el estudio respecto a las variables demográficas: sexo del bebé, nivel educativo y estrato socioeconómico $\left(\chi^{2}(1, N\right.$ $=93)=.001, p=0.970, X^{2}(4, N=67)=2.910$, $\left.p=0.573, X^{2}(2, N=93)=1.209, p=0.546\right)$.

\section{TABLA 1}

Descriptivos de las variables demográficas

\begin{tabular}{lccccc}
\hline \multicolumn{1}{c}{ Variable } & $N$ & $M$ & $D E$ & Mín. & Máx. \\
\hline \multicolumn{7}{c}{ Madres Canguro } \\
\hline Edad materna & 49 & 17.4 & 1.6 & 14 & 20 \\
Peso al nacer (g) & 49 & 1,960 & 328.4 & 1,120 & 2,867 \\
Edad gestacional & 49 & 34.5 & 2.2 & 28 & 38 \\
\hline \multicolumn{7}{c}{ Madres Cuidado } \\
\hline Regular \\
\hline Edad materna & 44 & 17.8 & 1.7 & 14 & 20 \\
Peso al nacer (g) & 44 & 3,012 & 376.9 & 2,400 & 3,900 \\
Edad gestacional & 44 & 38.9 & 1.5 & 34 & 42 \\
\hline
\end{tabular}

Fuente: elaboración propia. 
TABLA 2

Descriptivos sociodemográficos

\begin{tabular}{|c|c|c|}
\hline Variable & Frecuencia & Porcentaje \\
\hline \multicolumn{3}{|c|}{ Madres Cuidado } \\
\hline \multicolumn{3}{|c|}{ Canguro } \\
\hline \multicolumn{3}{|l|}{ Educación $n=37$} \\
\hline Primaria Incompleta & 1 & 2.7 \\
\hline Primaria Completa & 1 & 2.7 \\
\hline Bachillerato Incompleto & 14 & 37.8 \\
\hline Bachillerato Completo & 12 & 32.4 \\
\hline Educación Técnica & 9 & 24.3 \\
\hline \multicolumn{3}{|l|}{ Sexo $n=49$} \\
\hline Niños & 31 & 63.3 \\
\hline Niñas & 18 & 36.7 \\
\hline \multicolumn{3}{|l|}{ Estrato S-E $n=49$} \\
\hline Estrato 1 & 13 & 26.5 \\
\hline Estrato 2 & 12 & 24.5 \\
\hline Estrato 3 & 24 & 49.0 \\
\hline \multicolumn{3}{|c|}{ Madres Cuidado } \\
\hline \multicolumn{3}{|c|}{ Regular } \\
\hline \multicolumn{3}{|l|}{ Educación $n=30$} \\
\hline Primaria Incompleta & 1 & 3.3 \\
\hline Primaria Completa & 1 & 3.3 \\
\hline Bachillerato Incompleto & 16 & 53.3 \\
\hline Bachillerato Completo & 6 & 20.0 \\
\hline Educación Técnica & 6 & 20.0 \\
\hline \multicolumn{3}{|l|}{ Sexo $n=44$} \\
\hline Niños & 28 & 63.6 \\
\hline Niñas & 16 & 36.4 \\
\hline \multicolumn{3}{|l|}{ Estrato $S-E n=44$} \\
\hline Estrato 1 & 16 & 36.4 \\
\hline Estrato 2 & 8 & 18.2 \\
\hline Estrato 3 & 20 & 45.4 \\
\hline
\end{tabular}

Fuente: elaboración propia.

\section{Procedimiento}

La muestra de este estudio participó en una investigación más amplia que se ejecutó bajo un diseño cuasiexperimental longitudinal, multivariable de medidas repetidas (Arnau, 1995), con un pretest al nacimiento del bebé y tres mediciones adicionales hasta los 12 meses de edad corregida. La información en esta publicación se centra en las mediciones relacionadas con la calidad de cuidado materno a los 3 y 7 meses de edad del bebé.

Las madres participantes fueron contactadas y reclutadas entre las 24 y 72 horas posteriores al parto en cada uno de los hospitales. Las auxiliares de campo invitaban a las madres a participar voluntariamente, y se fueron reclutando para las dos condiciones de cuidado, canguro y cuidado regular, en la medida que los bebés iban naciendo. El grupo de madres adolescentes con bebés prematuros y participantes en el cuidado madre canguro fueron seleccionadas para participar en el estudio, si cumplían con las siguientes condiciones de elegibilidad: los bebés debían tener al nacer al menos 30 semanas de edad gestacional y no debían pesar menos de $1.201 \mathrm{~g}$; y los(as) niños(as) no podían tener ningún problema grave de salud. La auxiliar de campo le entregaba a la madre el consentimiento informado, y le preguntaba que si quería que lo leyeran juntas. Si la madre estaba de acuerdo con participar, la psicóloga buscaba a uno de los padres de la madre adolescente, le presentaba el documento y le pedía que lo firmara en compañía de su hija. Luego de la firma del consentimiento informado, la auxiliar iniciaba la recolección de información para la encuesta sociodemográfica.

Cuando los bebés tuvieron 3 meses de edad (corregida para los prematuros), las madres y el bebé realizaron una consulta pediátrica de control en el hospital. En esta visita, el comportamiento materno durante las interacciones de cuidado (alimentación, cambio de pañal, juego, vestir/desvestir) con su hijo/a fue registrado por un tiempo aproximado de 40 minutos, por dos observadores entrenados en las Escalas del Comportamiento Materno (Alzate, Carbonell, Posada \& Bustamante, 1999). Luego de finalizada la observación, cada 
uno de los observadores, de manera independiente, puntuó las escalas. Posteriormente a los 7 meses de edad (corregida para los prematuros), se llevó a cabo una visita domiciliaria de aproximadamente 2 horas con el fin de observar y describir el comportamiento de las madres, durante las interacciones de cuidado con su bebé. Después de la visita, cada observador(a) describió independientemente el comportamiento materno observado utilizando el Q-sort del Comportamiento Materno (Pederson \& Moran, 1995).

\section{Instrumentos}

La calidad del cuidado materno fue observada y descrita con las Escalas Cualitativas del Comportamiento Materno (Álzate et al., 1999) y con el Q-sort del Comportamiento Materno (MBQS) (Pederson \& Moran, 1995). Las Escala Cualitativas del Comportamiento Materno fueron diseñadas por Álzate et al. (1999). Este es un instrumento construido a partir de observaciones y registros etnográficos de 30 díadas mamá-bebé pertenecientes al estrato 3 , en las rutinas de cuidado diario (alimentación, baño, vestido y juego) en el hogar, en Bogotá (Posada et al., 2004). El instrumento consta de 11 subescalas: 1 . Prontitud de la respuesta, 2. Efectividad de la respuesta, 3. Consistencia comportamental, 4. Equilibrio entre las demandas del bebé y otras demandas, 5 . Equilibrio entre el cuidado físico y la interacción social y emocional con el bebé, 6. Disfrute de la interacción, 7. Fluidez interactiva, 8. Frecuencia del contacto físico, 9. Calidad del contacto físico, 10. Frecuencia de las verbalizaciones, 11 . Diversidad de las funciones en las comunicaciones verbales maternas. El comportamiento de las madres, en cada uno de los dominios, es calificado en una escala de 1-7. Las escalas del comportamiento materno muestran validez concurrente con el MBQS en un estudio realizado en Bogotá (Posada et al., 2004).

En este estudio dos observadores registraron y puntuaron las escalas independientemente $y$, en seguida, discutieron y negociaron sobre los ítems donde se presentaron discrepancias mayores a 2 puntos. Además, del puntaje de cada una de la escalas, se calculó un puntaje compuesto, promediando los puntajes obtenidos en cada uno de los dominios; éste puntaje fue el reflejo de la calidad del cuidado materno para cada madre de la muestra. Dichos puntajes, incluyendo el de la escala total, fueron utilizados en los análisis. La confiabilidad interobservador del puntaje total fue igual a un coeficiente de Kappa de .75.

El Q-sort del comportamiento materno, tiene 90 ítems basados en la conceptualización de Ainsworth acerca del cuidado temprano. El apoyo empírico y validación del MBQS han sido reportados por diversos estudios (Pederson, Gleason, Moran \& Bento, 1998; Pederson \& Moran, 1995, 1996; Pederson et al., 1990). El MBQS, ha sido traducido al español y la evidencia de su validez ha sido reportada por Posada et al. (1999, 2002). En el presente estudio, dos observadores realizaron una descripción del comportamiento materno en interacción con su bebé; sus descripciones fueron promediadas en una descripción compuesta. Un puntaje global de sensibilidad materna, que refleja la calidad de cuidado observado, fue obtenido correlacionando la descripción compuesta y el criterio teórico que describe a una madre óptimamente sensible (Pederson \& Moran 1995). La correlación entre estas dos descripciones es el puntaje de sensibilidad materna. Este puntaje fue usado para el análisis.

Observadores entrenados en la metodología $Q$ realizaron las visitas. Los observadores que puntuaron las escalas cualitativas del comportamiento materno son distintos a los que realizaron las descripciones con el Q-sort. El promedio de la confiabilidad interobservadores (Block, 1978) para el MBQS a los 7 meses de edad de los bebés, basado en el acuerdo interobservadores fue de 0.87 con un rango de puntajes entre 0.70 y 0.95 .

\section{Resultados}

La pregunta central de esta investigación examinó si existen diferencias significativas entre los dos grupos de madres adolescentes respecto a la calidad del cuidado materno, dependiendo del tipo de cuidado que prodigaron a su hijo(a), canguro o regular. Las estadísticas descriptivas de 
los puntajes totales de las escalas cualitativas de comportamiento materno a los 3 meses de edad y de los puntajes en sensibilidad materna a los 7 meses (edad corregida para los bebés prematuros), se presentan en la Tabla 3. Para cada una de las 11 escalas cualitativas, las estadísticas descriptivas se presentan en la Tabla 4.

TABLA 3

Descriptivos de Sensibilidad Materna

\begin{tabular}{|c|c|c|c|c|}
\hline Variable & $N \quad M$ & $D E$ & Mín. & Max. \\
\hline \multicolumn{5}{|c|}{ Grupo Canguro } \\
\hline \multicolumn{5}{|c|}{ Escalas Cualitativas } \\
\hline $\begin{array}{l}\text { Comportamiento } \\
\text { Materno } 3 \text { meses }\end{array}$ & $41 \quad 4.83$ & 1.09 & 1.90 & 6.50 \\
\hline $\begin{array}{l}\text { Sensibilidad Materna } 7 \\
\text { meses }\end{array}$ & $49 \quad 0.57$ & 0.29 & -0.21 & 0.85 \\
\hline \multicolumn{5}{|c|}{ Grupo Cuidado Regular } \\
\hline \multicolumn{5}{|c|}{ Escalas Cualitativas } \\
\hline $\begin{array}{l}\text { Comportamiento } \\
\text { Materno } 3 \text { meses }\end{array}$ & $45 \quad 4.81$ & 1.07 & 2.80 & 6.90 \\
\hline $\begin{array}{l}\text { Sensibilidad Materna } 7 \\
\text { meses }\end{array}$ & 440.59 & 0.31 & -0.41 & 0.85 \\
\hline
\end{tabular}

Fuente: elaboración propia.

TABLA 4

Descriptivos de Las Escalas Cualitativas del Comportamiento Materno

\begin{tabular}{|c|c|c|c|c|}
\hline Variable & M & DE & Min. & Max. \\
\hline \multicolumn{5}{|c|}{ Grupo Canguro } \\
\hline 1. Prontitud de la respuesta & 5.58 & 1.26 & 2.3 & 7.0 \\
\hline 2. Efectividad de la respuesta & 5.16 & 1.12 & 3.0 & 7.0 \\
\hline $\begin{array}{l}\text { 3. Consistencia comporta- } \\
\text { mental }\end{array}$ & 5.70 & .97 & 3.0 & 7.0 \\
\hline $\begin{array}{l}\text { 4. Equilibrio entre demandas } \\
\text { del bebé y otras demandas }\end{array}$ & 4.95 & 1.30 & 1.7 & 7.0 \\
\hline $\begin{array}{l}\text { 5. Equilibrio entre cuidado } \\
\text { físico y la interacción social y } \\
\text { emocional con el bebé }\end{array}$ & 4.43 & 1.53 & 1.0 & 7.0 \\
\hline 6. Disfrute de la interacción & 4.67 & 1.47 & 1.0 & 7.0 \\
\hline
\end{tabular}

\begin{tabular}{|c|c|c|c|c|}
\hline Variable & M & $D E$ & Min. & Max. \\
\hline 7. Fluidez interactiva & 5.06 & 1.36 & 1.0 & 7.0 \\
\hline $\begin{array}{l}\text { 8. Frecuencia del contacto } \\
\text { físico }\end{array}$ & 5.44 & 1.22 & 1.0 & 7.0 \\
\hline 9. Calidad del contacto físico & 5.21 & 1.29 & 1.7 & 7.0 \\
\hline $\begin{array}{l}\text { 10. Frecuencia verbaliza- } \\
\text { ciones }\end{array}$ & 3.65 & 1.80 & 1.0 & 7.0 \\
\hline $\begin{array}{l}\text { 11. Diversidad de las funcio- } \\
\text { nes en las comunicaciones } \\
\text { verbales maternas }\end{array}$ & 3.36 & 1.76 & 1.0 & 6.3 \\
\hline
\end{tabular}

\begin{tabular}{|c|c|c|c|c|}
\hline \multicolumn{5}{|c|}{ Grupo Cuidado Regular } \\
\hline 1. Prontitud de la respuesta & 5.08 & 1.28 & 3.0 & 7.0 \\
\hline 2. Efectividad de la respuesta & 5.18 & .98 & 2.0 & 7.0 \\
\hline $\begin{array}{l}\text { 3. Consistencia comporta- } \\
\text { mental }\end{array}$ & 5.50 & 1.26 & 3.0 & 7.0 \\
\hline $\begin{array}{l}\text { 4. Equilibrio entre demandas } \\
\text { del bebé y otras demandas }\end{array}$ & 4.90 & 1.19 & 2.0 & 7.0 \\
\hline $\begin{array}{l}\text { 5. Equilibrio entre cuidado } \\
\text { físico y la interacción social y } \\
\text { emocional con el bebé }\end{array}$ & 4.35 & 1.60 & 2.0 & 7.0 \\
\hline 6. Disfrute de la interacción & 4.71 & 1.18 & 2.0 & 7.0 \\
\hline 7. Fluidez interactiva & 5.11 & 1.15 & 2.0 & 7.0 \\
\hline $\begin{array}{l}\text { 8. Frecuencia del contacto } \\
\text { físico }\end{array}$ & 5.54 & 1.32 & 3.0 & 7.0 \\
\hline 9. Calidad del contacto físico & 5.28 & 1.27 & 2.3 & 7.0 \\
\hline $\begin{array}{l}\text { 10. Frecuencia verbaliza- } \\
\text { ciones }\end{array}$ & 3.68 & 1.79 & 1.0 & 7.0 \\
\hline $\begin{array}{l}\text { 11. Diversidad de las funcio- } \\
\text { nes en las Comunicaciones } \\
\text { verbales maternas }\end{array}$ & 3.64 & 1.78 & 1.0 & 7.0 \\
\hline
\end{tabular}

Fuente: elaboración propia.

Todas las escalas del comportamiento materno se asocian significativa y positivamente entre sí, en un rango que se distribuye entre rho $(86)=0.27$ a 0.93, $p<0.01$, bilateral. Así mismo, se observó una correlación positiva y significativa entre el puntaje total de las Escalas del Comportamiento Materno a los 3 meses de edad de los bebés y el puntaje global de sensibilidad materna medido por el Q-sort del Comportamiento Materno a los 7 meses de edad 
de los bebés (rho $(77)=0.32, p<0.01$, bilateral). En la muestra completa de madres adolescentes, todas las escalas de comportamiento materno, excepto dos: prontitud de la respuesta materna y efectividad de la respuesta materna, están significativa y positivamente asociadas con el puntaje del Q-sort del comportamiento materno, en un rango que se distribuye entre rho $(77)=0.27$ a 0.39 , con $p<0.05$ y 0.01 .

Los análisis de diferencias de medias entre los dos grupos de madres en cada uno de los instrumentos utilizados para evaluar la calidad del cuidado materno, indicaron que las diferencias entre los dos grupos de madres adolescentes en los puntajes de la escala total a los 3 meses y de la sensibilidad materna a los 7 meses (edad corregida para los bebés prematuros) no son significativas, $t$ (86) $=0.091, \mathrm{df}=84, p>0.05$, bilateral, $t$ (93) $=-0.387, \mathrm{df}=91, p>0.05$, bilateral.

Los análisis de varianza también indican que las diferencias entre los dos grupos (cuidado madre canguro y cuidado regular) en los puntajes para cada una de las escalas de comportamiento materno, no fueron significativas (prontitud de la respuesta materna $(F(1,84)=3.299, p>0.05)$, efectividad de la respuesta materna $(F(1,84)=$ $0.003, p>0.05)$, consistencia materna $(F(1,84)$ $=0.667, p>0.05)$, equilibrio en las demandas competitivas $(F(1,84)=0.033, p>0.05)$, equilibrio entre cuidados físicos y afectivos $(F(1,84)$ $=0.000, p>0.051)$, disfrute mutuo $(F(1,84)=$ $0.020, p>0.05)$, fluidez interactiva $(F(1,84)=$ $0.044, p>0.05)$, frecuencia del contacto físico $(F$ $(1,84)=0.115, p>0.05)$, calidad del contacto físico $(F(1,84)=0.052, p>0.05)$, frecuencia comunicativa $(F(1,84)=0.024, p>0.05)$ y diversidad de las funciones del lenguaje $(F(1,84)$ $=0.545, p>0.05)$.

\section{Discusión}

El cuidado madre canguro provee una experiencia temprana e intensa de contacto piel a piel entre la madre y su bebé prematuro. En este estudio, se evaluó si existían diferencias significativas en la sensibilidad materna de dos grupos de madres adolescentes de una población a riesgo, debido a sus características sociodemográficas, luego de la participación de uno de los grupos en el cuidado madre canguro.

Los resultados mostraron que no existen diferencias significativas en sensibilidad materna entre madres adolescentes con bebés a término, expuestos a cuidado materno y sin ninguna intervención en un grupo de alto riesgo y madres adolescentes provenientes del mismo sector de la población, quienes además tuvieron un bebé prematuramente y participaron en un programa madre canguro. Tampoco se encontraron diferencias significativas entre los dos grupos de madres respecto a sus comportamientos maternos específicos en sus interacciones con sus bebés a los tres meses de edad (por ejemplo, prontitud y efectividad de la respuesta materna, frecuencia y calidad del contacto físico, disfrute mutuo, equilibrio entre cuidado físico y cuidado social y afectivo) descritos con las escalas cualitativas.

El estresor adicional de la prematurez podría generar la expectativa de que las madres de bebés prematuros tuvieran más dificultades en implementar un patrón de cuidado considerado como sensible, debido a las dificultades que se han documentado en diversos estudios asociadas a la prematurez. Por ejemplo, bebés prematuros exhiben menor regulación emocional y menor nivel de atención y responsividad en las interacciones con sus padres. Esto, a su vez, hace más difícil la tarea de los padres de interpretar y responder apropiadamente a las señales y comunicaciones de su bebé (Brachfeld et al., 1980; Moran et al., 1992; Pederson \& Moran, 1995; Spiker et al., 1993). Sin embargo, los resultados no apoyan esa interpretación.

La falta de diferencias significativas entre los dos grupos de madres adolescentes provenientes de una población de alto riesgo, es consistente con la noción de que el cuidado madre canguro protege contra el impacto desfavorecedor que la prematurez pueda tener en la calidad del cuidado ofrecido por madres adolescentes pobres y de bajo nivel educativo. A pesar de contar con una condición estresante critica adicional (prematurez de su bebé), los puntajes de calidad de cuidado de 
madres con bebés prematuros fueron similares a los puntajes de las madres con bebés a término y cuidado regular, quienes no están sometidas al estrés relacionado con tener un bebé prematuro. Quizás, el participar en el cuidado madre canguro y tener la experiencia temprana e intensiva de contacto piel a piel, tuvo un papel protector contra cualquier efecto negativo extra que la prematurez añadiera a las ya difíciles circunstancias que las mamás de la muestra experimentan (Feldman et al., 2002; Feldman et al., 2003; Ortiz et al., 2006). El papel protector del cuidado madre canguro en madres adultas y adolescentes ha sido ilustrado también por Ortiz et al. (2006). Los resultados presentados en este estudio apuntan en la misma dirección.

Es importante tener en cuenta que las condiciones de vida de estas madres adolescentes de por sí, no son facilitadoras de comportamientos de cuidado que denoten sensibilidad (Posada et al., 1999). De hecho, aunque no se encontraron diferencias significativas entre los grupos, los puntajes de sensibilidad obtenidos por los dos grupos de madres adolescentes tienden a ser más bajos que los que son reportados para clases medias en Colombia, Canadá y Estados Unidos (Pederson \& Moran, 1995; Pederson \& Moran, 1996; Posada et al., 2002). Entre más baja la calidad de cuidado, menos óptima es la relación vincular entre padres y sus hijos/as. Así pues, aunque el cuidado madre canguro pudiera ofrecer un factor de protección, ciertamente no puede, ni pretende, contrarrestar el impacto negativo que otras condiciones de vida estresantes crónicas puedan tener en el desarrollo de las relaciones madre-bebé.

La asociación significativa, aunque moderada encontrada en la calidad del cuidado materno, al ser evaluada a los 3 y 7 meses de edad de los bebés, tanto en el contexto hospitalario como en el hogar, hace referencia a una cierta continuidad del comportamiento materno de cuidado, a pesar de las condiciones de inestabilidad que caracteriza a esta población de madres adolescentes. Es importante, señalar que ambos instrumentos poseen algunos dominios comportamentales maternos comunes, sin embargo, cada uno contribuye a la medición del constructo de la calidad del cuidado materno.

\section{Contribuciones y limitaciones}

La principal contribución de este estudio fue aportar evidencia que apoya la idea sobre el papel aparentemente protector del cuidado madre canguro en una muestra de madres adolescentes de alto riesgo. Entre las ventajas metodológicas se encuentra el haber realizado las observaciones del comportamiento materno en el medio familiar, lo cual incrementa la validez ecológica de la información recogida.

Entre las limitaciones se encuentra el hecho de que el estudio no se hizo con un diseño de muestras aleatorias; la muestra fue compuesta de madres adolescentes voluntarias. Importante, tampoco se realizaron varias mediciones del comportamiento materno en los primeros meses, utilizando los mismos instrumentos. Esto no solo no permitió obtener una mejor muestra de la conducta materna en interacción con el bebé, sino que no permitió evaluar de manera sistemática y similar la variabilidad que caracteriza el comportamiento de las mamás en las interacciones y en distintos contextos, así como los cambios intrasujeto e intersujeto a través del tiempo en estas muestras a riesgo, las cuales tienen condiciones de vida inestables. En otros estudios se ha observado que factores contextuales influyen en el comportamiento sensible materno (De Wolff \& van IJzendoorn, 1997; Egeland \& Farber, 1984; Thompson, Lamb, \& Estes, 1982; Vaughn, Egeland, Sroufe, \& Waters, 1979; Vondra, Hommerding, \& Shaw, 1999). Finalmente, el estudio no permitió evaluar cualquier efecto a más largo plazo que el cuidado madre canguro pudiera tener en la calidad del cuidado materno como lo sugiere la investigación de Ortiz et al. (2006).

En conclusión, las madres adolescentes de un sector vulnerable de la población que tuvieron un bebé prematuro y que participaron en el cuidado madre canguro, obtuvieron puntajes en la calidad del cuidado que ofrecieron a sus bebés, similares a los puntajes obtenidos por madres adolescentes del mismo sector de la población que tuvieron bebés a término. En la medida que el tener un bebé prematuro es un factor estresante que puede interferir en la habilidad de las madres para ser sensibles en el 
cuidado de sus bebés, la similitud de los puntajes de los dos grupos sugiere que el cuidado madre canguro brinda un papel protector.

\section{Referencias}

Agencia Pandi. (2009). Un "retraso" con múltiples consecuencias. Recuperado el 25 de noviembre, 2009 de www.agenciapandi.org

Alzate, G., Carbonell, O. A., Posada, G. \& Bustamante, M. R. (1999). Escalas cualitativas del comportamiento materno. Manuscrito no publicado, Pontificia Universidad Javeriana, Bogotá, Colombia.

Anisfeld, E., Casper, V., Nozyce, M. \& Cunningham, N. (1990). Does infant carrying promote attachment? An experimental study of the effects of increase physical contact on the development of attachment. Child Development, 61, 1617-1627.

Arnau, J. (1995). Diseños Longitudinales aplicados a las ciencias sociales y del comportamiento. México: Limusa.

Block, J. (1978). The q-sort method: In personality assessment and psychiatric research. California: Consulting Psychologists Press.

Brachfeld, S., Goldberg, S. \& Sloman, J. (1980). Parentinfant interaction in free play at 8 and 12 months: Effects of prematurity and immaturity. Infant Behavior and Development, 3, 289-305.

Centro Latinoamericano Salud y Mujer, AC. (2009). Día Nacional para la Prevención del Embarazo No Planificado en Adolescentes: Mensaje de la Secretaría de Salud. México. Recuperado el 11 noviembre, 2009 de http://www. celsam. org/home/noticia Home

Charpak, N., Figueroa de Calume, Z. \& Ruiz-Peláez, J. G. (2000). "The Bogotá Declaration on Kangaroo Mother Care": Conclusions at the Second International Workshop on the Method. Acta Paediatrica, 89, 1137-1140.

Charpak, N., Ruiz-Peláez, J. G. \& Figueroa de Calume, Z. (1996). Current knowledge of Kangaroo mother intervention. Current Opinion in Pediatrics, 8, 108-112.

Charpak, N., Ruiz-Peláez, J. G. \& Figueroa de Calume, Z. (2000). Commentary: Humanizing Neonatal Care. Acta Paediatrica, 89, 501-502.
Charpak, N., Ruiz-Peláez, J. G., Figueroa de Calume, Z. \& Charpak, Y. (2001). A Randomized, Controlled Trial of Kangaroo Mother Care: Results of Follow-Up at 1 Year of Corrected Age. Pediatrics, 108, 1072-1079.

Child Trends Data Bank. (2004). Teen Births. Recuperado el 3 junio, 2006 de http://www.childtrendsbank. org/indicators/13TeenBirth.cfm

Child Trends Data Bank. (2008). Teen Births. Recuperado el 11 noviembre, 2009 de http://www. childtrendsbank.org/indicators/13TeenBirth.cfm

Cattaneo, A., Davanzo, R., Bergman, N. \& Charpak, N. (1998). Kangaroo mother care in low-income countries. Journal of Tropical Pediatrics, 44, 279 . 282.

Darroch, J. E., Singh, S., Frost, J. J. \& the Study Team. (2001). Differences in teen pregnancy rates among five developed countries: The roles of sexual activity and contraceptive use. Family Planning Perspectives, 33, 244-250 y 281.

De Wolff, M. S. \& van IJzendoorn, M. H. (1997). Sensitivity and attachment: A meta-analysis on parental antecedents of infant attachment. Child Development, 68, 571-591.

Egeland, B. \& Farber, E. A. (1984). Infant-mother attachment: factors related to its development and changes over time. Child Development, 55, 753-771.

Feldman, R., Eidelman, A. I., Sirota, L. \& Weller, A. (2002). Comparison of skin-to-skin (Kangaroo) and traditional care: Parenting outcomes and preterm infant development. Pediatrics, 110, 16-26.

Feldman, R., Weller, A., Sirota, L. \& Eidelman, A. I. (2002). Skin-to-skin contact (kangaroo care) promotes self-regulation in premature infants: Sleep-wake ciclicity, arousal modulation, and sustained exploration. Developmental Psychology, 38, 194-207.

Feldman, R., Weller, A., Sirota, L. \& Eidelman, A. I. (2003). Testing a family intervention hypothesis: The contribution of mother-infant skin to skin contact (Kangaroo care) to family interaction, proximity and touch. Journal of Family Psychology, 17, 94-107.

Figueroa de Calume, Z. \& Charpak, N. (1996). Una técnica Colombiana de cuidado ambulatorio del 
niño prematuro o de bajo peso al nacer. Tribuna Medica, 93, 191-200.

Haskett, M. E., Johnson, C. \& Miller, J. (1994). Individual differences in risk of child abuse by adolescent mothers: Assessment in the perinatal period. Journal of Child Psychology and Psychiatry, 35, 461-476.

Lutzker, J. R., Van Hasselt, V. B., Bigelow, K. M., Greene, Brandon, F. \& Kessler, M. L. (1998). Child abuse and neglect: Behavioral research, treatment, and theory. Aggression and Violent Behavior, 3, 181-196.

Moore, M. \& Brooks-Gunn, J. (2002). Adolescent parenthood. In M. Borstein (Ed.). Handbook of parenting.Vol.3 Being and becoming a parent (pp. 173214). Mahwah, NJ: Lawrence Erlbaum Associates. Moran, G., Pederson, D. R., Pettit, P. \& Krupka, A. (1992). Maternal sensitivity and infant-mother attachment in a developmentally delayed sample. Infant Behavior and Development, 15, 427-442.

Ogi, S., Fukada, M., Takahashi, T., Akiyama, T., Morichuchi, H., Nugent, J., Brazelton, T. B. et al. (2002). The effects of Kangaroo care on neonatal neurobehavioral organization, infant temperament and development in healthy low-birth-weight infants over the first year of life: The Nagasaki university hospital study. AB INITIO INTERNATIONAL Winter 2001-2002. Disponible en http://www.brazelton-institute.com/ abinitio2002/art1.html

Ortiz, J. A., Borré, A., Carrillo, S. \& Gutiérrez, G. (2006). Relación de apego en madres adolescentes y sus bebés canguro. Revista Latinoamericana de Psicología, 38, 71-86.

Pederson. D. R. \& Moran, G. (1995). A categorical description of infant-mother relationships in the home and its relation to Q-sort measures of infantmother interaction. In E. Waters, B. E. Vaughn, G. Posada \& K. Kondo-Ikemura (Eds.), Care giving, cultural, and cognitive perspectives on secure-base behavior and working models: New growing points of attachment theory and research (pp. 111-132). Monographs of the Society for Research in Child Development, 60 (2-3, Serial No. 244).

Pederson, D. R. \& Moran, G. (1996). Expressions of the attachment relationship outside of the strange situation. Child Development, 67, 915-927.
Pederson, D. R., Moran, G., Sitko, C., Campbell, K., Ghesquire, K. \& Acton, H. (1990). Maternal sensitivity and the security of infant-mother attachment: A q-sort study. Child Development, 61, 1974-1983.

Pederson, D. R., Bento, S., Chance, G. W., Evans, B. \& Fox, A. M. (1987). Maternal emotional responses to preterm birth. American Journal of Orthopsychiatry, 57, 15-21.

Pederson, D.R., Gleason, K. E., Moran, G. \& Bento, S. (1998). Maternal attachment representation, maternal sensitivity, and the infant-mother attachment relationship. Developmental Psychology, 34, 925-933.

Posada, G., Carbonell, O. A, Alzate, G. \& Plata, S. J. (2004). Through Colombian lenses: Ethnographic and conventional analyses of maternal care and their associations with secure base behavior. Developmental Psychology, 40, 508-518.

Posada, G., Gao, Y., Wu, F., Posada, R., Tascon, M. et al. (1996). The secure base phenomenon across cultures: Children's behavior, mothers' preferences, and experts' concepts. In E. Waters, B. E. Vaughn, G. Posada \& K. Kondo-Ikemura (Eds.), Caregiving, cultural, and cognitive perspectives on secure base behavior and working models: New growing points of attachment theory and research (pp. 27-48). Monographs of the Society for Research in Child Development, 60 (2-3 Serial No. 244).

Posada, G., Jacobs, A., Carbonell, O. A., Alzate, G., Bustamante, M. R. \& Arenas, A. (1999). Maternal Care and Attachment Security in Ordinary and Emergency Contexts. Developmental Psychology, 35, 1379-1388.

Posada, G., Jacobs, A., Richmond, M. K., Carbonell, O. A., Alzate, G., Bustamante, M. R. \& Quiceno, J. (2002). Maternal caregiving and infant security in two cultures. Developmental Psychology, 38, 67-78.

Profamilia. (2005). Salud Sexual y Reproductiva en Zonas Marginales: Situación de las Mujeres Desplazadas [Resumen de Prensa]. Bogotá: USAID - PROFAMILIA.

PubMed (2003). Teenage pregnancy, a worldwide problem. Recuperado el 11 de noviembre, 2009, de http://www.ncbi.nlm.nih.gov/pubmed 
Scholl, T. O., Hediger, M. L. \& Belsky, D. H. (1994). Prenatal care and maternal health during adolescent pregnancy: A review and meta-analysis. Journal of Adolescent Health Care, 15, 444-456.

Spiker, D., Fergurson, J. \& Brooks-Gunn, J. (1993). Enhancing maternal interactive behavior and child social competence in low birth weight, premature infants. Child Development, 64, 754-768.

Stevens-Simons, C. \& Nelligan, D. (1998). Strategies for identifying and treating adolescents at risk for maltreating their children. Aggression and Violent Behavior, 3, 197-217.

Tessier, R., Cristo, M., Velez, S., Giron, M., Figueroa de Calume, Z., Ruiz, J.G., Charpak, Y. \& Charpak, N. (1998). Kangaroo mother care and the bonding hypothesis. Pediatrics, 102, 1-8.

Tessier, R., Cristo, M. B., Velez, S., Giron, M., Nadeau, L., Figueroa de Calume, Z. et al. (2003). Kangaroo mother care: A method for protecting high-risk low-birth-weight and premature infants against developmental delay. Infant Behavior and Development Journal, 26, 384-397.

Thompson, R. S., Lamb, M. E. \& Estes, D. (1982). Stability of infant-mother attachment and is relationship to changing life circumstances in an unselected middle-class sample. Child Development, 53, 144-148.
Torres Ruiz, G. (2009). Insuficiente el acceso a información y servicios de salud sexual y reproductiva. América Latina - Aumenta el embarazo en adolescentes. Recuperado el 11 de noviembre, 2009, de http: //www.alterinfos. org/spip.php

Vaughn, B., Egeland, B., Sroufe, L. A. \& Waters, E. (1979). Individual differences in infant-mother attachment at twelve and eighteen months: Stability and change in families under stress. Child Development, 50, 971-975.

Vondra, J. I., Hommerding, K. D. \& Shaw, D. S. (1999). Stability and change in infant attachment in a lowincome sample. In J. Vondra \& D. Barnett (Eds.), Atypical patterns of infant attachment: Theory, research, and current directions (pp. 119-144). Monographs of the Society for Research in Child Development, 64 (3, Serial No.258).

World Health Organization. (2007). Action to address adolescent pregnancy. Recuperado el 29 de mayo, 2008 de http://www.who.int/ child_adolescent_ health/news/2007_10/en/print.html

World Health Organization. (2009). Adolescent pregnancy. Recuperado el 11 noviembre, 2009 de http://www.who.int/making_pregnancy_safer/ topics/adolescent pregnancy/ en /print.html 
